\title{
Choroidal Excavation in Eye with Normal Tension Glaucoma
}

\author{
Kazunobu Asao ${ }^{a, b}$ Takeshi Morimoto ${ }^{b}$ Atsuko Nakada ${ }^{a}$ \\ Yoshimi Kawasaki ${ }^{\mathrm{a}}$ \\ ${ }^{\mathrm{a}}$ Department of Ophthalmology, Takarazuka Daiichi Hospital, Hyogo, and ${ }^{\mathrm{b}}$ Department of \\ Ophthalmology, Osaka University Graduate School of Medicine, Osaka, Japan
}

\section{Key Words}

Choroidal excavation · Humphrey Field Analyzer · Inner/outer segment junction line · Normal tension glaucoma Optical coherence tomography

\begin{abstract}
Purpose: To report the case of an eye with normal tension glaucoma and a choroidal excavation. Methods: This is an observational case report. Results: A 59-year-old woman with normal tension glaucoma had a choroidal excavation in the left eye. Her best-corrected visual acuity and intraocular pressure were within normal limits and had been stable for 5 years. Fundus examination showed a small white lesion inferior to the macula and a nerve fiber layer defect at the inferior edge of the optic disc. Humphrey Field Analyzer (HFA) showed visual field defects corresponding to the nerve fiber layer defect with C30-2, and a central scotoma superior to the macula with C10-2. Optical coherence tomography (OCT) showed a 150- $\mu \mathrm{m}$ deep choroidal excavation. Disruptions of the IS/OS line were detected only in the area inferior to the choroidal excavation. During the 5 months of follow-up, her best-corrected visual acuity remained at 1.0 and the IOP ranged from 12 to $14 \mathrm{~mm} \mathrm{Hg}$ in the left eye. The fundus and OCT images did not deteriorate and the choroidal excavation did not enlarge. Conclusions: The disruption of the inner/outer segment (IS/OS) line was detected only at the area surrounding the choroidal excavation. OCT examinations are useful in assessing the area of the residual IS/OS line, and HFA can be used to estimate the residual central visual field.


Asao et al.: Choroidal Excavation in Eye with Normal Tension Glaucoma

\section{Introduction}

An anomalous excavation of the choroid was first reported by Jampol et al. [1] in an eye with good visual acuity and a normal appearance of the overlying retina without any evidence of a posterior staphyloma or scleral ectasia. It has been reported that Asian women have a high incidence of choroidal excavations, and other studies have suggested that a choroidal excavation may be a congenital anomaly of the macula regions [2]. The choroidal excavations may be associated with central serous chorioretinopathy (CSC), secondary choroidal neovascularization, or polypoidal choroidal vasculopathy [2-5]. However, the long-term follow-up of the patients with choroidal excavation has not yet been fully reported.

The improvements in the resolution of optical coherence tomography (OCT) instruments has made it possible to obtain more detailed information about the different layers of the retina and the choroid [6]. One of the defects detected by these instruments is a choroidal excavation. Choroidal excavations have been divided into either the conforming type in which there is no separation between the photoreceptor tips and the retinal pigment epithelial (RPE), or the non-conforming type in which the photoreceptor tips appear to be detached from the underlying RPE with an intervening hyporeflective space, presumably filled with subretinal fluid [3].

OCT is useful for diagnosing patients with glaucoma because the changes in the macular thickness can be followed and correlated with changes in the visual field, especially in the central 10 degrees [7]. Because of the higher incidence of visual field defects in the central region, it is recommended that intensive testing of the central 10 degrees of the visual field (10-2 strategy) should be performed on patients with normal tension glaucoma (NTG) [8]. We describe a case of NTG with a choroidal excavation.

\section{Case Report}

A 59-year-old woman complained of metamorphopsia for 5 years when using her left eye. Her family history and her personal medical history were not significant. Her bestcorrected visual acuity (BCVA) was 1.2 in both eyes, and the intraocular pressure (IOP) was $14 \mathrm{~mm} \mathrm{Hg}$ in both eyes. Her refractive errors (spherical equivalent) were -2.25 diopters (D) OD and -2.75 D OS, and an Amsler chart examination showed distorted vision in the superior central field. Her color vision was also abnormal. Slit-lamp examination showed no abnormalities in both eyes, and a gonioscopy showed that the angles were open with the presence of normal iris processes. Left fundus examination showed a small white lesion inferior to the macula and a nerve fiber layer defect (NFLD) at the lower edge of the optic disc and the right fundus was normal (fig. 1a, b). The cup-to-disc ratio was 0.7 for the left eye, and Humphrey Field Analyzer (HFA) showed visual field defects corresponding to the NFLD with C30-2 (fig. 1c) and a scotoma superior to the macula with C10-2 (fig. 1d).

The patient was diagnosed with NTG, and HFA was performed periodically to observe the changes of the visual field.

In our hospital, the OCT apparatus was introduced only 6 months ago. To investigate the white lesion in more detail, we performed several OCT examinations of the macular area (Scanning laser ophthalmoscopic image) (fig. 2a). OCT image (layer thickness map) showed the region of the IS/OS line defects (fig. 2b). When we performed OCT examinations for the first time, a 150- $\mu \mathrm{m}$ deep choroidal excavation and a highly reflective RPE band, following the contour of the choroidal excavation, were detected. The vertical OCT image showed the 
Asao et al.: Choroidal Excavation in Eye with Normal Tension Glaucoma

same disruptions of the inner/outer segment (IS/OS) line as indicated by 2 colored arrows (fig. 2c). We performed OCT examinations at the patient's last visit. The OCT images did not show the spreading of the choroidal excavation and the disruptions of the IS/OS line remained unchanged (fig. 2d).

Unfortunately, the patient was allergic to contrast media, and we could not perform an angiography.

During the follow-up period of 5 years, her BCVA remained at 1.0, and the IOP ranged from 12 to $14 \mathrm{~mm} \mathrm{Hg}$ in the left eye. For her 6-month follow-up, OCT images showed that the IS/OS line remained disrupted, but it did not worsen. The mean depth of the choroidal excavation was $150 \mu \mathrm{m}$ with a range of $145-155 \mu \mathrm{m}$. After 5 years, her visual acuity and visual fields remained unchanged.

\section{Discussion}

It is known that choroidal excavations are associated with CSC and secondary choroidal neovascularization [2-5]. However, we could not perform an angiography because of the patient's allergies and we did not have earlier data of her choroid, but there were no signs that she had had CSC. In our case, if she did not have NTG, the choroidal excavation would have been performed earlier but because of our focus on NTG, it led us to overlook a possible excavation. We recommend that when a patient suffers from a central scotoma, intensive testing of the central 10 degrees of the visual field (10-2 strategy) should be performed in addition to the OCT examination.

Our case had the non-confirming type of choroidal excavation, and the OCT examinations showed an area of presumed hyporeflective subretinal fluid separating the photoreceptor OS tips from the underlying retinal pigment epithelium over the choroidal excavation. We found that a disruption of the IS/OS line was only at the area inferior to the choroidal excavation. We suppose that this depended on the traction from the inferior area. This lead to the vulnerability of the eye itself and the reactivity to the changes such as the IOP, blood pressure, trauma, and intracranial diseases. We need to seek for the systemic disease that led to these changes and know the features of the choroidal excavation.

The separation of the photoreceptor OS tips caused the abnormality between the photoreceptors OS and RPE [3]. The decrease in the length of the IS/OS line has been reported to be significantly correlated with a decrease in retinal sensitivity and visual acuity [9]. The IS/OS line in retinitis pigmentosa patients is lost from the periphery toward the fovea with a progression of the disease. Our patient complained of a visual field defect and distorted vision. Measuring the length of the residual IS/OS line is useful in estimating the residual central visual function [10]. During the follow-up examinations, OCT showed a continuous reduction of the IS/OS line at the lower part of the choroidal excavation. It is possible that the choroidal excavation may enlarge over time and therefore result in further ischemia to the overlying retina, atrophic changes, and visual disturbances [3]. To observe the changes of the visual field, HFA may be useful (as it was in our case). In addition, HFA with C10-2 rather than C30-2 might give more information about eyes with a choroidal excavation. It is essential to perform OCT and HFA examinations and know whether the visual field is maintained. We think it would be useful to report the 5-year follow-up.

Our findings did not provide evidence of a direct connection between the NTG and the choroidal excavation. Therefore, we presumed that NTG and the choroidal excavation occurred in the same patient by chance, but further studies are necessary. 
Asao et al.: Choroidal Excavation in Eye with Normal Tension Glaucoma

\section{Conclusion}

We reported a case of NTG with choroidal excavation. Our new finding was that the defect of the IS/OS line was detected only at the area inferior to the choroidal excavation. OCT examinations were helpful in assessing the area of the residual IS/OS line, and HFA can be useful in estimating the residual central visual function. We recommend that HFA with 10-2 should be performed to examine the changes of the visual field.

\section{References}

1 Jampol LM, Shankle J, Schroeder R, Tornambe P, Spaide RF, Hee MR: Diagnostic and therapeutic challenges. Retina 2006;26:1072-1076.

-2 Margolis R, Mukkamala SK, Jampol LM, Spaide RF, Ober MD, Sorenson JA, Gentile RC, Miller JA, Sherman J, Freund KB: The expanded spectrum of focal choroidal excavation. Arch Ophthalmol 2011;129:1320-1325.

-3 Wakabayashi Y, Nishimura A, Higashide T, Ijiri S, Sugiyama K : Unilateral choroidal excavation in the macula detected by spectral-domain optical coherence tomography. Acta Ophthalmol 2010;88:e87-e91.

4 Katome T, Mitamura Y, Hotta F, Niki M, Naito T: Two cases of focal choroidal excavation detected by spectral-domain optical coherence tomography. Case Rep Ophthalmol 2012;3:96-103.

5 Abe S, Yamamoto T, Kirii E, Yamashita H: Cup-shaped choroidal excavation detected by optical coherence tomography: a case report. Retinal Cases Brief Rep 2010;4:373-376.

-6 Srinivasan VJ, Monson BK, Wojtkowski M, Bilonick RA, Gorczynska I, Chen R, Duker JS, Schuman JS, Fujimoto JG: Characterization of outer retinal morphology with high-speed, ultrahigh-resolution optical coherence tomography. Invest Ophthalmol Vis Sci 2008;49:1571-1579.

7 Lee KS, Lee JR, Na JH, Kook MS: Usefulness of macular thickness derived from spectral-domain optical coherence tomography in the detection of glaucoma progression. Invest Ophthalmol Vis Sci 2013;54:19411949.

-8 Thonginnetra 0, Greenstein VC, Chu D, Liebmann JM, Ritch R, Hood DC: Normal versus high tension glaucoma: a comparison of functional and structural defects. J Glaucoma 2010;19:151-157.

-9 Aizawa S, Mitamura Y, Hagiwara A, Sugawara T, Yamamoto S: Changes of fundus autofluorescence, photoreceptor inner and outer segment junction line, and visual function in patients with retinitis pigmentosa. Clin Experiment Ophthalmol 2010;38:597-604.

-10 Rangaswamy NV, Patel HM, Locke KG, Hood DC, Birch DG:: A comparison of visual field sensitivity to photoreceptor thickness in retinitis pigmentosa. Invest Ophthalmol Vis Sci 2010;51:4213-4219. 
Case Reports in

Ophthalmology
Asao et al: Choroidal Excavation in Eye with Normal Tension Glaucoma

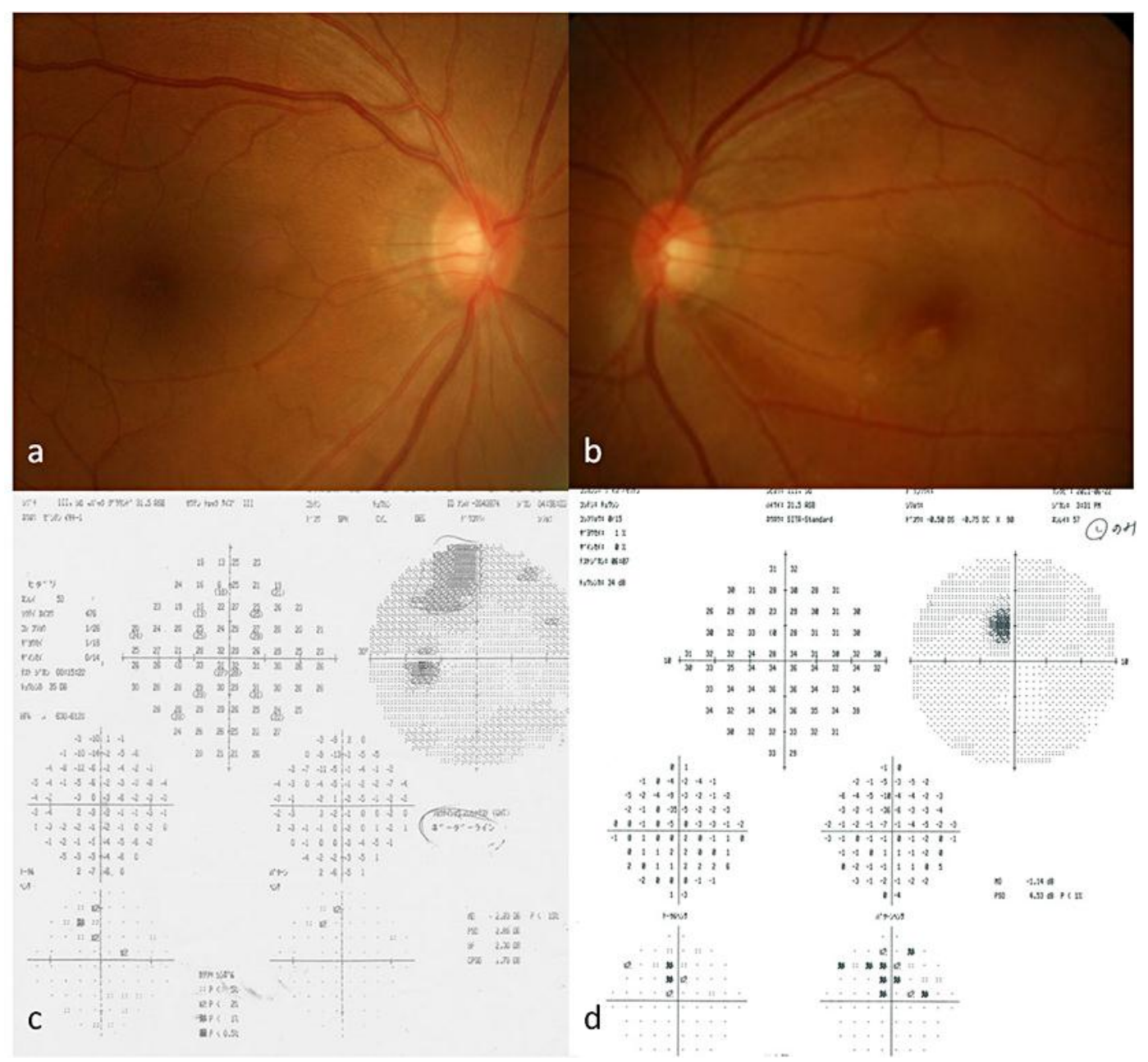

Fig. 1. Findings in a patient with NTG combined with choroidal excavation. a The right fundus photograph was normal. $\mathbf{b}$ Left fundus photograph showing a small white lesion inferior to the macula and nerve fiber layer defect at the lower edge of the optic disc. c HFA with C10-2 showing a relative sensitivity loss corresponding to the choroidal excavation. $\mathbf{d}$ HFA with $\mathrm{C} 30-2$ showing visual field defects corresponding to the NFLD. 


\section{Case Reports in \\ Ophthalmology}

\begin{tabular}{l|l}
\hline Case Rep Ophthalmol 2014;5:144-149 & \\
\hline DOI: 10.1159/000363131 & $\begin{array}{l}\text { ○ 2014 S. Karger AG, Basel } \\
\text { www.karger.com/cop }\end{array}$ \\
\hline
\end{tabular}

Asao et al.: Choroidal Excavation in Eye with Normal Tension Glaucoma

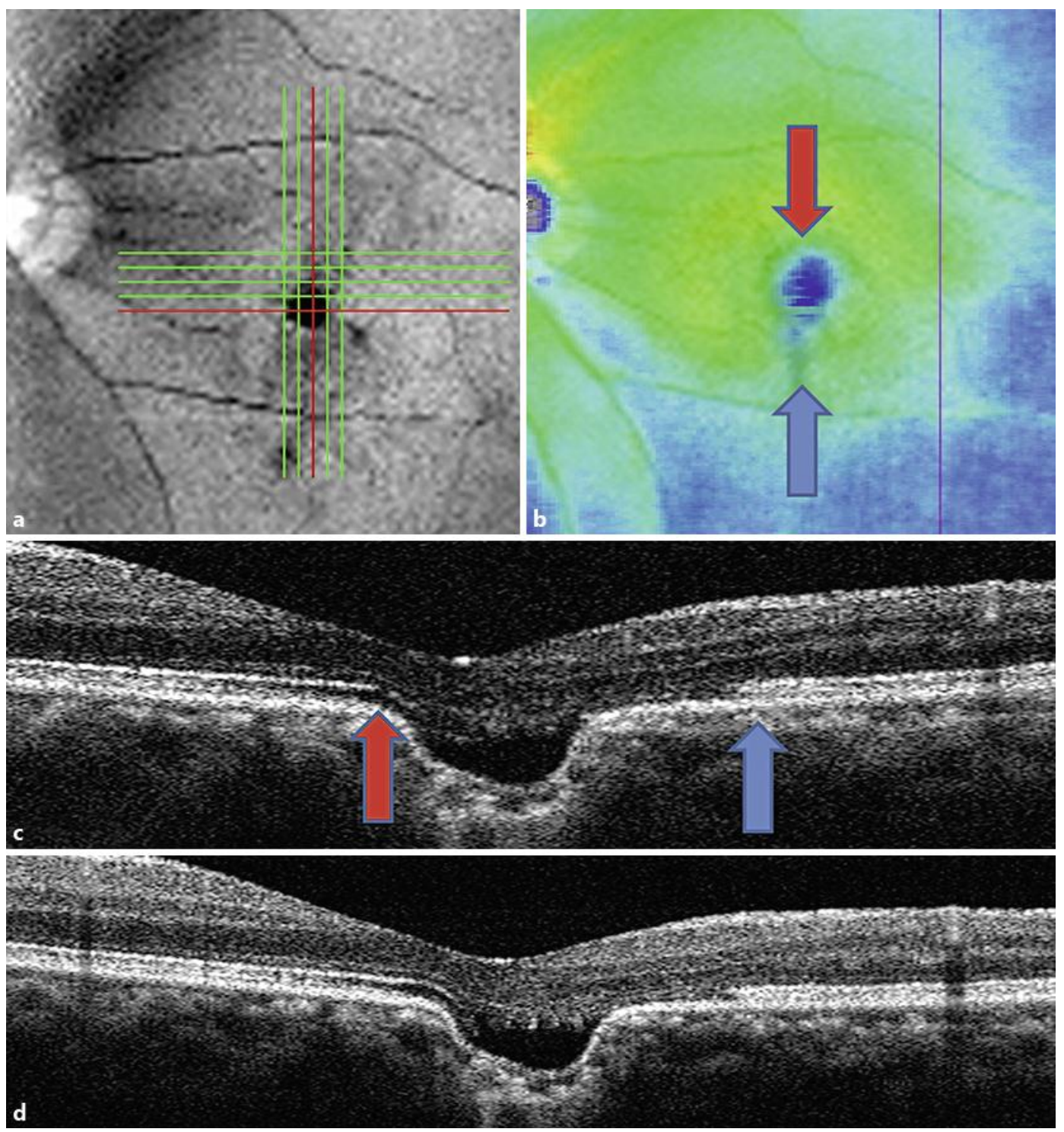

Fig. 2. OCT findings of our patient. a, b OCT examinations using RS 3000 (Nidek, Gamagori, Japan) were performed. Scanning laser ophthalmoscopic image and layer thickness map show the region of the IS/OS line defects ( 2 colored arrows. Red = upper; blue = lower). c Initial vertical OCT image of the left eye showing the same defect of the IS/OS line by two colored arrows from the choroidal excavation to the inferior area. $\mathbf{d}$ At the patient's 6-month follow-up, the vertical OCT image showed the same defect of the IS/OS line. 